Revue

de I'histoire des religions
Revue de l'histoire des religions

$4 \mid 2015$

Figures de Noé de Gilgamesh au Coran

\title{
« Noé » dans les sources mésopotamiennes
}

"Noah" in Mesopotamian sources

Jean-Jacques Glassner

\section{(2) OpenEdition}

Journals

Édition électronique

URL : http://journals.openedition.org/rhr/8460

DOI : 10.4000/rhr.8460

ISSN : 2105-2573

Éditeur

Armand Colin

Édition imprimée

Date de publication : 1 décembre 2015

Pagination : 487-498

ISBN : 978-2-200-93012-7

ISSN : 0035-1423

Référence électronique

Jean-Jacques Glassner, « « Noé » dans les sources mésopotamiennes », Revue de l'histoire des religions [En ligne], 4 | 2015, mis en ligne le 01 décembre 2018, consulté le 19 avril 2019. URL : http:// journals.openedition.org/rhr/8460 ; DOI : 10.4000/rhr.8460 


\section{« Noé » dans les sources mésopotamiennes}

Dans les sources mésopotamiennes, le survivant du déluge, précurseur du Noé biblique, s'appelait Ziusudra, Atrahasîs ou Ûta-napishtî. Le premier nom signifiait "Vie de jours prolongés», le second "Le Supersage", le troisième "J'ai trouvé ma vie». Ce qui le distinguait de tous les autres êtres humains, c'était le destin d'être immortel qui lui était réservé au sortir de l'épreuve. Le franchissement du déluge était une forme d'épreuve. La Mésopotamie connaissait deux héros civilisateurs, Sargon et Ziusudra/Atrahasîs/Ûta-napishtî. Leurs expositions respectives n'eurent pas même signification. L'un était élu avant l'épreuve, l'autre après. Pour le premier, on a affaire à un rite de probation; pour le second on est en présence d'un rite d'institution, mais qui diffère des rites de passage.

\section{"Noah" in Mesopotamian sources}

In Mesopotamian sources, the surviver of the flood had three names: Ziusudra, Atrahasîs or Ûta-napishtî. The first name meant "Life of long days", the second "The Supersage", the third "I have found my life". What distinguished him from other human beings was his status as an immortal being, which was offered to him after the flood. The crossing of the flood was a kind of ordeal. Mesopotamia had two civilizing heroes, Sargon of Akkade and Ziusudra/Atrahasîs/ Uta-napishtî. Their respective exposures did not have the same meaning. The one was chosen before the ordeal, the other after it. For the first, we are confronted with a rite of probation, for the second with a rite of institution, but a rite which differs from an ordinary rite of passage. 
Les lettres mésopotamiennes connaissent le déluge. Le mot qui le désigne est amaru en sumérien, abûbu en akkadien. Quatre sources, principalement, le documentent : le mythe d'Atrahasîs, connu principalement par une copie paléo-babylonienne du XVII ${ }^{\mathrm{e}}$ siècle, de la main du scribe Ipiq-Aya ${ }^{1}$; d'autres copies plus tardives viennent en compléter certaines lacunes; le mythe tel qu'il est conté dans la tablette XI de l'Épopée de Gilgamesh, dans la version de Ninive $\left(\mathrm{VII}^{\mathrm{e}} \text { siècle }\right)^{2}$; le récit sumérien du déluge, documenté par une copie unique, apparemment du $\mathrm{XVI}^{\mathrm{e}}$ siècle, mais dont la langue est défaillante au point qu'elle fait penser à une traduction effectuée à cette même époque de l'akkadien en mauvais sumérien ${ }^{3}$; le récit de Bérose, en grec, à l'usage d'un public grec, et qui date de l'époque hellénistique ${ }^{4}$.

\section{DifFÉRENTS DÉLugeS}

Le déluge est un motif tardif et peu exploité dans la littérature sumérienne. Le mot apparaît au $\mathrm{XXI}^{\mathrm{e}}$ siècle avant notre ère. amaru signale alors un phénomène météorologique ou une arme redoutable entre les mains de la déesse Inanna. Il est au cœur du genre littéraire de la lamentation, où il est fait référence à la colère des dieux à l'égard des villes dont ils sont les divinités poliades, une colère qui désigne ces villes pour la destruction. Il fait alors allusion à un ouragan balayant tout sur son passage, car le phénomène est essentiellement céleste. Ce n'est guère avant le $\mathrm{Xx}^{\mathrm{e}}$ siècle que les théologiens ou les mythographes d'Isin conviennent de le situer dans le temps du mythe, c'est-à-dire à l'origine, tout en le créditant d'une portée universelle. Environ un siècle plus tard, à la charnière des $\mathrm{XIX}^{\mathrm{e}}$ et $\mathrm{XVIII}^{\mathrm{e}}$ siècles, les historiens l'introduisent dans la trame de

1. Wilfred George Lambert et Allan R. Millard, Atra-has $\bullet$. The Babylonian Story of the Flood, Oxford, Clarendon, 1969.

2. Andrew R. George, The Babylonian Gilgamesh Epic, Oxford, University Press, 2 vol., 2003.

3. Miguel Civil in W.L. Lambert et A.R. Millard, voir note 1.

4. Stanley M. Burstein, The Babyloniaca of Berossos, Sources and Monographs 1/5, Malibu, Undena Publications 1978. 
l'histoire ${ }^{5}$, le déluge étant susceptible de répétition, chaque époque pouvant faire l'expérience d'un avatar du cataclysme premier. Ainsi, le mascaret du peuple Guti envoyé par Enlil pour détruire Akkadé ou Sumer et Ur est-il comparé à un déluge, un ouragan violent qu'accompagnent des averses de grêle ${ }^{6}$. L'introduction longue et circonstanciée de la chronique royale babylonienne montre que c'est définitivement chose faite dès la fin de l'époque paléo-babylonienne ${ }^{7}$. Dans la Bible, l'épisode de la tour de Babel est une métaphore qui évoque un nouveau déluge avec le mascaret humain qui se répand sur la terre entière.

Le déluge est davantage un motif akkadien, comme le laisse entendre, déjà, la liste des œuvres qui le décrivent. Les sources paléo-babyloniennes montrent qu'il est élaboré à partir de plusieurs traditions distinctes. L'une est sumérienne, on l'a vu, elle décrit les tempêtes destructrices de villes. Une seconde concerne l'existence de rois antédiluviens dont des listes énumèrent les noms. Ces listes s'accordent toutes sur un point : la dernière ville royale d'avant le déluge fut Shuruppak et le dernier roi fut Ziusudra, le fils et successeur d'Ubar-Tutu'. Une troisième tradition, enfin, nourrit le concept de déluge, celle du sommeil des dieux contrarié par le vacarme de l'humanité. Le mot akkadien vacarme, hubûru, dit de façon métaphorique l'activité créatrice de l'humanité industrieuse et l'indépendance de cette humanité qui a hérité de l'esprit frondeur de la classe des dieux laborieux.

Dans un premier temps, le déluge est conçu comme un phénomène atmosphérique venu du ciel, à l'instar des sources sumériennes. Tel et le cas du déluge dans la version d'Atrahasîs copiée par Ipiq-Aya : Enlil, le roi des dieux, ayant confié le ciel à Adad, le dieu de l'orage, celui-ci déverse des torrents d'eau sur la terre. Le dieu Adad est donc à la manœuvre. « Au premier point du jour, est-il écrit, monta de l'horizon une noire nuée dans laquelle tonnait Adad (...) Nergal arracha les étais des vannes célestes, et Ninurta se précipita pour

5. Jean-Jacques Glassner, Mesopotamian Chronicles, Writings from the Ancient World 19, Atlanta, Society of Biblical Literature, 2004, p. 117 sq. ; ce texte ne figure que dans les versions de l'époque d'Isin ou de Larsa.

6. Piotr Michalowski, The Lamentation over the Destruction of Sumer and Ur, Winona Lake, Eisenbrauns, 1989, p. 40 : 75-84.

7. J.-J. Glassner, voir note 5 , ch. V.

8. J.-J. Glassner, voir note 5, p. 57s. 
faire déborder les barrages d'en haut, tandis que les Anunnaki brandissaient leurs torches et incendiaient de leur embrasement le pays tout entier. Adad étendait dans le ciel son silence de mort, réduisant en ténèbres tout ce qui avait été lumineux ! (...) Personne ne voyait plus personne. Les foules n'étaient plus discernables dans cette trombe d'eau (...)». «Six jours et sept nuits durant, bourrasques, pluies battantes, ouragans et déluge ne cessèrent de saccager la terre ». Rappelons, pour mémoire, que, dans ce récit, le déluge est précédé par deux tentatives avortées de destruction de l'humanité par les dieux : une épidémie et une sécheresse.

Le texte de l'épopée de Gilgamesh présente le même récit et il s'agit encore d'un phénomène céleste, une formidable tempête faite de bourrasques et de pluies battantes.

Dans l'intervalle, le mythe s'enrichit d'une imagerie redondante. L'idée se fait jour d'une humanité bruyante parce que nombreuse et hostile, de ce fait dangereuse pour les dieux sur lesquels elle pourrait l'emporter, comme les petits dieux laborieux l'avaient fait, autrefois, avant la création de l'homme, selon le mythe d'Atrahasîs. Mais il est plus important, la description du cataclysme prend une nouvelle consistance avec le thème de la jonction des eaux d'en bas et d'en haut. Ce thème apparaît clairement exprimé dans le mythe d'Erra ${ }^{9}$ (IX ${ }^{\mathrm{e}}$ siècle) où Marduk annonce que, s'il quitte sa demeure, le lien de l'univers se défera, les eaux souterraines monteront et dévasteront la terre, en même temps qu'une tempête obscurcira le jour en ténèbres et occultera les étoiles. Comment ne pas se souvenir, à ce propos, de la description de la sécheresse dans le mythe d'Atrahasîs, une description qui en est l'exact opposé : "Qu'Adad réduise à rien les pluies, et que la crue, en bas, n'arrive plus de sa source !»; il en résulta que, « tandis qu'en bas, la crue n'arriva plus de sa source, la terre, de son sein, ne mit plus rien au monde».

On relève, en passant, que l'épisode du lâcher d'oiseau, tel que le narre la version ninivite de l'Epopée de Gilgamesh, est absent, en l'état des manuscrits, de la version paléo-babylonienne d'Atrahasîs (il pourrait figurer dans une lacune du texte). Par son vol, qui sépare les eaux d'en haut de celles d'en bas, l'oiseau ne recréet-il pas le cosmos en ébauchant la différenciation de ses éléments constitutifs?

9. Luigi Cagni, L'Epopea di Erra, Studi Semitici 34, Université de Rome, 1969. 
Quoi qu'il en soit, le déluge est la négation de la création, le retour à l'obscurité et au silence. Sa description même induit l'existence, antérieurement à la création, d'un monde indifférencié, une image que retiendront les auteurs de la Genèse.

Le mythe d'Erra offre de son côté une autre conception du cataclysme universel, où le déluge est absent. Marduk y évoque en ces termes une première colère et un premier départ de sa demeure : « Le lien de l'univers se défit. Le ciel ayant été ébranlé, la position des étoiles changea sans qu'elles pussent reprendre leur place. Le monde infernal ayant bronché, le produit des sillons s'amenuisa, rendant désormais difficile la subsistance. Le lien de l'univers défait, la nappe souterraine baissa et le niveau des eaux descendit ».

Cette vision concorde avec les assertions d'un autre mythe babylonien, «La Glorification de Marduk $»^{10}$. Son auteur, la composition date du XII ${ }^{\mathrm{e}}$ siècle, met en scène le dieu de Babylone en train, non pas de créer, mais de mettre en ordre le cosmos. Pour instaurer une métrique du ciel et pour réguler le mouvement cyclique, prédéterminé et uniforme, des étoiles et des astres, il installe sa propre étoile, Nêberu, la planète Jupiter au moment où elle devient visible au-dessus de l'horizon oriental. À partir de cette position, pour organiser la géographie céleste, il calcule celles d'Enlil et d'Éa avec les étoiles qui les rendent manifestes. Elles constituent toutes les trois autant de bornes jalonnant le parcours des corps célestes et elles empêchent tout risque de déviation dans leurs trajectoires, ce que l'auteur du mythe appelle toute « faute » ou toute « négligence », autant d'actes que les dieux Lune et Soleil sont chargés de réprimer. Ce passage du mythe est commenté par les scribes du roi d'Assyrie Asarhaddon (VII ${ }^{\mathrm{e}}$ siècle) en ces termes ${ }^{11}:$ " Les étoiles du firmament se rendirent dans leurs positions, elles empruntèrent leur chemin véridique et abandonnèrent leur chemin non véridique ».

Quant au récit sumérien du déluge, il reprend à son compte la chronologie mise en place par les auteurs de la Chronique de la monarchie une : à l'époque antédiluvienne, les mêmes villes royales se succèdent dans le temps avec, à leur tête, les mêmes rois. Le

10. Philippe Talon, Enûma Elish, State Archives of Assyria Cuneiform Texts IV, Helsinki, The Neo-Assyrian Text Corpus Project, 2005.

11. Earl Leichty, The Royal Inscriptions of Esarhaddon, King of Assyria (680-669 BC), The Royal Inscriptions of the Neo-Assyrian Period 4, Winona Lake, Eisenbrauns, 2011, passim. 
dernier de ces monarques, Ziusudra de Shuruppak, est informé par son dieu de l'imminence du désastre.

\section{Un SeUl héros, mais Plusieurs noms. Ses qualités}

Le survivant du déluge est un personnage unique, quoiqu'affublé de trois noms, Ziusudra est un nom sumérien qui signifie «Vie de jours lointains/prolongés », une façon très mésopotamienne de dire une vie éternelle. Il est roi par sa naissance, mais il est aussi un sage éminent, l'auteur d'un recueil de maximes ${ }^{12}$. Au sortir de l'épreuve, il semble toujours exercer la royauté puisqu'il dispose toujours de la prérogative royale de construire des temples ${ }^{13}$. Utta-napishtî est le nom qui lui est attribué dans l'épopée de Gilgamesh où il est également qualifié de rûqu, « le lointain ». Il signifie «J'ai trouvé ma vie » et se veut la traduction akkadienne de Ziusudra; rûqu est une allusion au sumérien sudra, « lointain ». En persan médiéval on retrouve le nom transcrit 'tnbysh ou At(a)nabîsh. Le troisième nom, Atrahasîs, « Le Supersage », apparaît davantage comme un surnom ou un qualificatif que comme un véritable anthroponyme. Il insiste sur la profondeur de la sagesse du personnage, entendons par ce mot son extrême dévotion.

Doué d'intelligence, il sait se faire architecte, car il apprend vite la leçon du dieu Enki/Éa qui lui commande de construire un bateau et dessine pour lui une épure sur le sol. La source sumérienne est avare de détails, elle nous apprend simplement qu'il construit un «cargo », má.gur ${ }_{4}$ gur $_{4}$. Bérose se contente de rappeler que le navire fait cinq stades de long sur deux de large. L'auteur de l'Atrahasîs, pour sa part, se montre hésitant. Un éclat de tablette propose une description sommaire d'un bateau rond dont on ignore s'il s'inscrivait dans une sphère. La source principale parle d'un bateau équilatéral où sont aménagés plusieurs ponts superposés et qui doit être hermétiquement clos et calfaté. Mais on ne comprend pas bien s'il doit le couvrir avec une bâche ou autre chose, le mot urpatu, "nuage », pouvant désigner une tente, une bâche ou un taud. La version ninivite de l'Épopée de Gilgamesh en offre la

12. Bent Alster, The Instructions of Shuruppak, Copenhague, Akademisk Forlag, 1974.

13. Gilgamesh I 42. 
description la plus achevée. Le bateau forme un cube de 90 mètres d'arête, avec une surface de 8100 mètres carrés par face. Il est divisé en sept étages et chaque étage en neuf compartiments.

Tout le monde l'a compris tant les études sont nombreuses à ce sujet, le bateau, à l'instar de l'arche dans la Genèse, est une représentation réduite du cosmos. Nous avons donc affaire à une reproduction d'un cosmos cubique en format réduit. Il est remarquable que les mesures de la tour de Babylone coïncident avec celles du navire tel qu'il est décrit dans l'Épopée de Gilgamesh. $\mathrm{Ne}$ mesure-t-elle pas, à sa base, selon une tablette cunéiforme conservée au Musée du Louvre, 90 mètres de côté, le premier étage étant divisé en neuf espaces ? N'est-elle pas composée de sept étages pour atteindre 90 mètres de hauteur, le dernier étage étant lui-même divisé en neuf compartiments ? Mais il est une différence de taille, elle ne forme pas un cube mais une tour dont les étages sont toujours plus petits à mesure que l'on s'élève en altitude. La tour n'est donc pas une reproduction du microcosme, elle en est l'armature, l'ossature sans laquelle le cosmos ne pourrait tenir ensemble. Elle tient de l'arbre cosmique qui plongeait ses racines dans le tréfonds du monde inférieur et dont les frondaisons rejoignaient le ciel supérieur ${ }^{14}$.

Mais ce qui distingue tout particulièrement le héros, c'est le destin qui lui est réservé au sortir de l'épreuve. Les dieux lui accordent en effet le statut d'être immortel. Comme le souligne, par exemple, le texte sumérien, ils le gratifient de $t i$ dingir.gin, « la vie comme (celle d')un dieu », ou zi.da-rí dingir.gin ${ }_{7}$, "la vie éternelle comme (celle d')un dieu ». De ce moment, il est installé à Dilmun, au-delà des mers, l'actuel Bahrayn.

Or, le héros du déluge est le seul être humain à quitter son statut de mortel pour acquérir l'immortalité. Gilgamesh lui-même, la figure la plus emblématique de la royauté, doit y renoncer. Adapa ${ }^{15}$, le sage par excellence, n'y parvient pas davantage. On se souvient de sa mésaventure. Il est le plus illustre des sept sages de la tradition, celui auquel les dieux ont accordé la connaissance des principes de l'univers ; une légende rapporte qu'il brisa les ailes du vent du Sud.

14. J.-J. Glassner, «L'Etemenanki, armature du cosmos», Nouvelles Assyriologiques Brèves et Utilitaires 2002/1, 14.

15. Shlomo Izre'el, Adapa and the South Wind, Winona Lake, Eisenbrauns, 2001. 
Or, en commettant cet acte, n'a-t-il pas agi à l'instar des dieux ? Ceux-ci décident donc de le mettre à l'épreuve. L'ayant convoqué, ils s'arrangent pour lui faire savoir qu'ils lui tendent un piège : ils lui offriront, en guise de repas d'hospitalité, de l'eau de mort et de la nourriture de mort et non point, comme le veut la coutume, de l'eau-de-vie et de la nourriture de vie, promesses d'immortalité. Refusant le don d'eau et de nourriture de vie qui lui était, en réalité, présenté, Adapa tombe dans le piège qui lui était tendu et retourne mortel sur terre. La leçon est clairement assénée : Adapa, tel un nouvel Edipe, s'est trouvé confronté à son destin déjà accompli ! La morale de l'histoire se trouve peut-être dans cette réprimande qu'Hélios adresse à Phaéton lequel, prétendant conduire le char solaire, avait provoqué un incendie cosmique : "Ton destin est d'un mortel, ton ambition d'un immortel ».

\section{LE SOUVENIR DE RITES SOCIAUX ?}

Pour ce qui concerne le héros, l'épisode du déluge a toute l'apparence d'une étape lors de l'accomplissement d'un rite de passage ou d'adoption. Un rite de passage accompagne, pour un individu ou un groupe, un changement de statut; le seuil qui est franchi opère une division entre les membres de la société qui ont subi le rite et tous les autres. Il comporte trois étapes successives au cours desquelles la notion de seuil est centrale : les préliminaires, les liminaires et les post-liminaires, trois termes qui sont dérivés du latin limen, «seuil », et qui peuvent se décliner autrement : séparation, marginalité, agrégation. Le néophyte quitte l'environnement social dans lequel il vit, il passe par un ailleurs absolu qui permet son isolement et l'accomplissement des transformations des valeurs; cette disjonction spatiale est une nécessité absolue ; enfin, il réintègre la société où il tient une place qui n'était pas la sienne antérieurement.

À distance d'Arnold Van Gennep et de Victor Turner, avec Pierre Bourdieu ${ }^{16}$, on préfère parler de rite d'institution. Cet auteur s'interroge en effet. En mettant l'accent sur le passage de l'enfance à l'adolescence, n'a-t-on pas évacué la vraie question qui est posée,

16. Pierre Bourdieu, «Les rites comme actes d'institution», in Pierre Centlivres et Jacques Hainard, éds, Les Rites de passage aujourd'hui, Lausanne, L'Âge d'Homme, 1986, p. 206-215. 
celle de la fonction et de la signification sociales de la ligne de seuil dont le rite autorise la transgression ? Ce n'est pas tant le passage qui importe que le seuil et le symbolisme qu'il véhicule. Il opère une division entre ceux qui sont justiciables du rite et les autres. Le rite consacre donc une différence essentielle dans la société.

La cérémonie est aussi l'occasion de la transmission d'un savoir secret. Elle implique la participation de trois types d'acteurs, les novices, les initiateurs et les non-initiés. Elle institue donc une différence entre ceux qui sont justiciables de l'initiation et les autres. Avec la transmission de connaissances secrètes qui sont d'autant plus précieuses qu'elles sont difficilement accessibles, le passage initiatique comporterait également une transformation en profondeur des impétrants, ce savoir secret impliquant à son tour trois types d'actants, celui qui détient le secret, celui à qui il le transmet et celui qui en est exclu. N'est-il pas révélé au héros un secret sur lequel il doit garder le silence, un secret qu'Enki/Éa dévoile à une paroi de roseau : un déluge va détruire toutes les villes et anéantir la race humaine ; pour échapper au cataclysme, il doit construire un bateau où il embarquera le jour venu. Le secret, comme le souligne à juste titre Jacques Derrida ${ }^{17}$ à propos du sacrifice d'Abraham et du silence que Dieu impose à ce dernier, « est la forme de l'épreuve, le signe de l'obéissance absolue » aux puissances invisibles.

L'épreuve que subit le héros ne saurait se réduire, cependant, à un banal rite de passage, car, dans la société, il s'est opéré, préalablement au déluge, une rupture de l'ordre et le rôle du héros consiste, précisément, à supprimer l'aliénation et rétablir l'ordre perturbé. Il est donc un sujet agi, obéissant aux forces invisibles. Il maintient vivant le lien qui unit le haut et le bas, les dieux et les hommes. Et telle sera sa fonction au sortir de l'épreuve, avec l'offrande du sacrifice. Sa récompense sera l'immortalité, mais avec le complément indispensable, son exclusion de la société des hommes, sans être véritablement admis, pour autant, dans la société des dieux. En termes greimassiens, on dira que le dieu remplit la fonction de destinateur, il charge le héros d'une mission de salut, préserver le genre humain qu'il a lui même créé ; il investit le héros du rôle de destinataire; l'accomplissement de sa mission sera récompensé par la vie éternelle.

17. Jacques Derrida, Sur Parole, Paris, Éditions de l'Aube, 1999, p. 77. 
La Mésopotamie a deux héros prométhéens, l'un est le survivant du déluge, l'autre est Sargon d'Akkadé, le fondateur du premier royaume qui unifie toute la Mésopotamie sous la férule d'un seul. Le rôle du premier s'arrête au sortir de l'épreuve, après la présentation d'un sacrifice destiné à restaurer le lien vertical de l'univers un moment rompu. Le rôle du second, exposé lors de sa naissance dans un couffin au gré d'un cours d'eau, commence, au contraire, au sortir de la nacelle dans laquelle il avait été exposé. Il crée un ordre universel de type monarchique. Des rapports étroits unissent entre elles ces deux figures qui sont conduites l'une et l'autre à triompher du même type d'épreuve : l'exposition dans un coffre flottant. La Bible le souligne avec assez de force qui appelle d'un même mot, têbâh, «arche » ou «nacelle », le bateau de Noé et le couffin de Moïse.

Sargon $^{18}$ est exposé à sa naissance, il n'est pas investi d'une fonction, il est exposé pour échapper à la mort consécutivement à une faute commise par sa mère. Celle-ci est une prêtresse êntu, précisément cette catégorie de femmes à laquelle, selon le mythe d'Atrahasîs, il est formellement interdit d'avoir des enfants. D'un mot, il y a rupture de l'ordre universel et cette rupture est consécutive au non-respect d'un interdit, lui-même mobile de l'exposition. Son élection par la divinité suit l'exposition et ne la précède pas, à l'opposé de Ziusudra, d'Atrahasîs ou d'Ûta-napishtî.

L'histoire de Sargon n'est pas celle du héros du déluge, elle en est la suite : rétablir l'ordre politique et social universel qui avait été rompu. La légende le concernant est composée d'épisodes successifs marquant les étapes de la conquête du pouvoir et faisant de Sargon l'archétype du fondateur. Elle débute par une présentation succincte du héros et de ses titres, affirmation au moyen d'une proposition nominale intemporelle d'une réalité objective et d'un ordre immuable fondé sur l'autorité royale. Suit alors le récit proprement dit. Mis au monde en secret, Sargon est abandonné au gré d'un cours d'eau dans un coffre entièrement clos. L'exposition précède son adoption par le puiseur d'eau Aqqi, lequel lui enseigne son propre métier de jardinier. Dans les procédures d'adoption, cette exposition hors du monde socialisé signifie l'extinction des droits

18. J.-J. Glassner, «Le récit autobiographique de Sargon», Revue d'Assyriologie 82, 1988, p. 1-11. 
des parents, préalable indispensable à la création d'un droit nouveau au profit de l'adoptant. L'expression consacrée est « la mère avait jeté l'enfant dans la gueule du chien » ou « dans le fleuve », autant de lieux d'où l'adoptante le retire.

Mais le récit ne s'arrête pas au seul motif de l'adoption. L'image du nouveau-né inclus dans un coffre, quppu, dit une nouvelle naissance ; l'aube d'une nouvelle vie : comment ne pas la rapprocher des motifs de la barque, eleppu, représentation du ventre de la mère, et du voyage en mer loin du regard du soleil accompli par l'enfant qui va naître ${ }^{19}$. Il s'agit aussi d'un rituel d'initiation, l'enfant étant exposé au néant, au chaos illustré par les eaux du fleuve. La cérémonie d'initiation est donc une répétition de la naissance. Mais Sargon est appelé à une élection et un accroissement de pouvoir. Il a pour rôle de rétablir un ordre perturbé. Introduit dans un espace mythique fondamentalement différent de l'espace socialisé, il accomplit dans ce but une série de performances, exposition puis éducation, dont la gradation le conduit à une issue victorieuse. L'ordre cosmique étant rétabli, l'enfant victorieux ne témoigne-t-il pas aussi d'une mère immaculée ? Le rapport à la mère génitrice n'est donc pas éteint.

\section{Conclusion}

Sargon et Ziusudra/Ûta-napishti/Atrahasîs sont deux héros civilisateurs. L'exposition de l'un et l'autre, apparemment rapprochées dans la Bible, n'a pas même signification. Les deux personnages s'opposent et se complètent. L'un est élu avant l'épreuve, l'autre après. Pour le second, on est en présence d'un rite d'institution, mais qui diffère des rites de passage. Pour le premier, on a affaire à un rite de probation. La structure narrative est la suivante : il existe un ordre social ; il y a rupture de cet ordre ; le héros se détache de la société, se charge d'une mission, celle de supprimer l'aliénation et de rétablir l'ordre perturbé ; dans le cas de l'adulte exposé, le héros est un sujet agi ; dans le cas de l'enfant exposé, il prend les décisions qui s'imposent après l'épreuve ; sa réussite est due à sa propre ingéniosité qui annonce son élection par

19. Nieck Velduis, A Cow of Sîn, Groningue, Styx, 1991. 
une déesse ; dans tous les cas, le héros victorieux est récompensé pour sa réussite; le lieu où se déroule l'épreuve est essentiel : c'est un au-delà qui assure la solitude du héros et l'accomplissement des transformations des valeurs.

jjglassner@wanadoo.fr 